\title{
CAD-/CAM-Rekonstruktion nach ablativer Chirurgie im Gesichtsschädelbereich
}

\author{
Moritz A. Birkelbach, Christian Lohse, Henning Hanken, Max Heiland, \\ Alexander Gröbe
}

\section{Zusammenfassung}

Aufgrund der persistierend hohen Inzidenz von Tumoren im Kopf-Hals-Bereich besteht diesbezüglich ein hoher Bedarf an Therapieoptionen. Trotz Fortschritten in der onkologischen Tumortherapie ist eine ausgedehnte operative Resektion bei diesen Tumoren weiterhin in vielen Fällen unumgänglich. Anhand eines klinischen Beispiels möchten wir in diesem Artikel die Fortschritte der operativen Therapie, insbesondere der CAD-/CAM-basierten (CAD/CAM: Computer Aided Design/Manufacturing) plastischen Rekonstruktion, erläutern.

\section{CAD/CAM Reconstructions after Ablative Surgery in the Oro-maxillo-facial Region}

As malignant tumors in the maxillofacial region are still common, therapeutic regimens must be optimised. Even though there have been improvements in recent years, surgery is still the "gold standard" for most malignant tumours in this area. Here we describe progress in modern CAD/CAM (CAD/ CAM: Computer Aided Design/Manufacturing) planning and operating systems, as applied during resection and reconstruction of patients diagnosed with malignant tumours in the oromaxillo-facial area.

\section{Einleitung}

Bei weltweit ca. 600000 Neuerkrankungen pro Jahr mit steigender Tendenz gehören maligne Entartungen, insbesondere das Plattenepithelkazinom der Mundhöhle, zu den 10 häufigsten malignen Tumoren des Menschen [1]. Trotz intensiver Forschung, vor allem im molekulargenetischen Bereich der Tumorbiologie, konnte über die letzten Jahre keine wesentliche Verbesserung der Prognosen betroffener Patienten erzielt werden. Somit nimmt die operative Therapie und plastische Defektrekonstruktion einen hohen Stellenwert ein und kann heutzutage bereits als Goldstandard bei intraoralen wie auch extraoralen Malignomen des Gesichtsschädelbereich angesehen werden [2-4].

Während bei intraoralen Tumoren des Oberkiefers nach Resektion bez. der Defektdeckung häufig zufriedenstellende

OP-JOURNAL 2016; 32: 237-240

(c) Georg Thieme Verlag KG Stuttgart · New York DOI http://dx.doi.org/10.1055/s-0042-121515 ästhetische und funktionelle Ergebnisse durch eine Anfertigung von individuellen Prothesen und Implantaten erreicht werden können, ist eine Defektdeckung im Unterkiefer in den meisten Fällen nur durch eine mikrovaskuläre Transplantation von autologem Gewebe möglich [5]. Kontinuitätsdefekte des Unterkiefers bedeuten für den Patienten häufig massive Beeinträchtigungen der Nahrungsaufnahme, der Kommunikation und des äußeren Erscheinungsbilds. Langstreckige Defekte werden heute in den meisten Fällen mit mikrochirurgischen Transplantaten gedeckt, wobei das Ausmaß des begleitenden Weichgewebedefizits die Auswahl der Entnahmeregion beeinflusst [6]. Ein häufig verwendetes Transplantat ist das Fibulatransplantat, welches mit oder ohne Hautinsel gehoben werden kann. Insbesondere eine relativ konstante Anatomie am Entnahmeort mit wenigen Varianzen des versorgenden Gefäßes und ein verhältnismäßig großes Lumen der Gefäße mit einer einfach zu erlernenden Entnahme des Transplantats machen es zu einem der am häufigsten eingesetzten mikrovaskulären knöchernen Transplan- tate [7]. Alternative Entnahmeregionen sind das Becken und das Schulterblatt.

Nach ablativer Chirurgie sind mikrochirurgische autologe Gewebstransplantate der „Goldstandard“ in der Defektrekonstruktion.

\section{Rekonstruktion eines Unterkiefer- defekts mittels CAD-/CAM-geplanter Fibulatransplantation anhand eines klinischen Beispiels}

Als operatives Verfahren kommen sowohl das zweizeitige als auch das einzeitige Verfahren in Betracht. Während beim zweizeitigen Verfahren der Tumor zunächst nur entfernt und in einer 2. Operation der Defekt gedeckt wird, erfolgt beim einzeitigen Verfahren die Resektion und plastische Rekonstruktion in einer Sitzung (nach Erhalt tumorfreier Schnellschnitte intraoperativ). Eine exakte anatomische, funktionelle und ästhetische Rekonstruktion ist in jedem Fall vonnöten. Diesbezüglich konnten mittels neuer CAD-/CAM-Technik (Computer Aided Design/Manufacturing) große Fortschritte in der plastisch-rekonstruktiven Chirurgie verzeichnet werden. Dabei handelt es sich um eine computergestützte 3-dimensionale Planungsmethode, die es ermöglicht, anhand von Computertomografie $(\mathrm{CT})$, digitaler Volumentomografie (DVT) oder Magnetresonanztomografie (MRT) gewonnenen Datensätzen im Vorfeld der eigentlichen Operation eine virtuelle Resektion durchzuführen, Schablonen für die Defektrekonstruktion zu erstellen und individualisierte Rekonstruktionsplatten anzufertigen. Darüber hinaus lassen sich inbesondere bez. der Resektion intraoraler Defekte dentale Rehabilitationen unmitelbar präoperativ simulieren und bereits intraoperativ berücksichtigen. Dies möchten wir anhand eines klinischen Beispiels an dieser Stelle demonstrieren.

Die computerbasierte CAD-/CAM-Rekonstruktion nach ablativer Chirurgie 


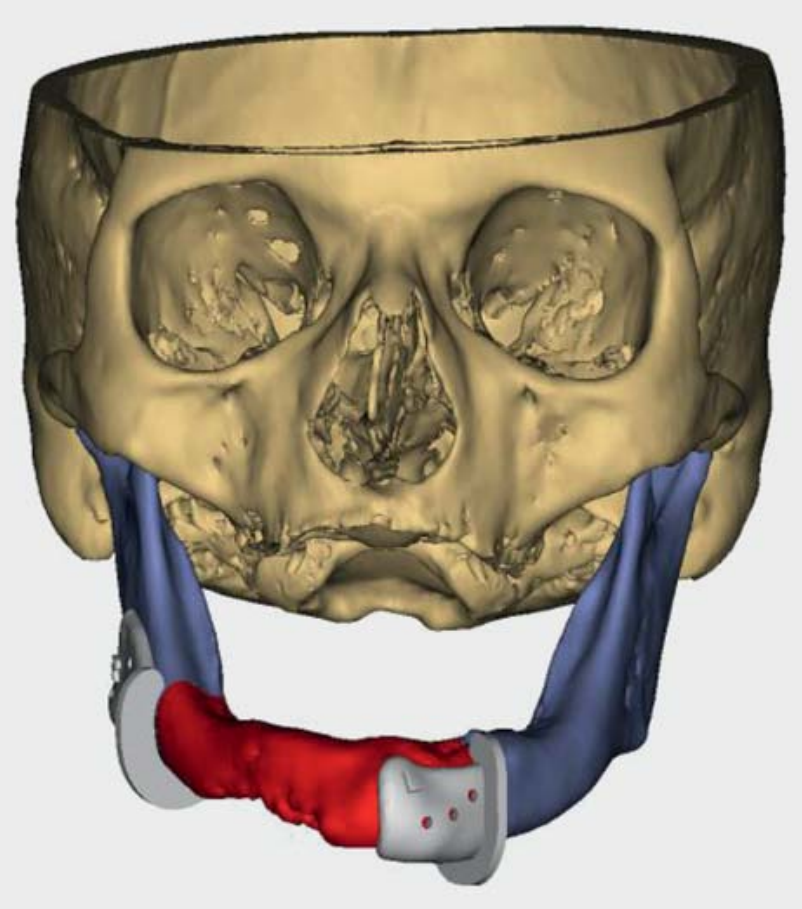

Abb. 1 Virtuelles 3-D-Modell zur Planung der kontinuitätsunterbrechenden Unterkieferresektion rechts (rot) mit entsprechenden Resektionsschablonen (hellgrau).

im Gesichtsschädelbereich beinhaltet eine Planungsphase, eine Produktionsphase und die Operationsphase. Die Planungsphase beginnt mit einer Erstellung eines defektorientierten hochauflösenden Scans der Kopf-Hals-Region. Dies kann mittels CT, MRT oder DVT erfolgen. Da entsprechende Bildgebung insbesondere bei malignen Erkrankungen Teil des klinischen Stagings zwecks Erstellung einer exakten Tumorformel ist, kann dabei auf die entsprechenden Scans zurückgegriffen werden. Es entsteht somit keine zusätzliche Strahlenbelastung für den Patienten. Zusätzlich zur lokoregionären Bildgebung ist in jedem Fall ein hochauflösender Scan der Entnahmeregion unerlässlich, damit hinterher in der Produktionsphase eine exakte Berechnung und Dimensionierung des $\mathrm{zu}$ entnehmenden Gewebes durchgeführt werden kann und entsprechende Schablonen hergestellt werden können. Bei dem Scan der Entnahmeregion gilt in den meisten Fällen eine hochauflösende CT als Mittel der Wahl, da diese, gekoppelt mit einer angiografischen Darstellung, eine Darstellung der Gefäßverhältnisse ermöglicht und somit dem Operateur wichtige Informationen liefert bei der Wahl der Präparation der Gefäße, die dann in der Operationsphase als Blutversorgung für das mikrochirurgische Transplantat dienen. Somit können postoperative Minderperfusionen

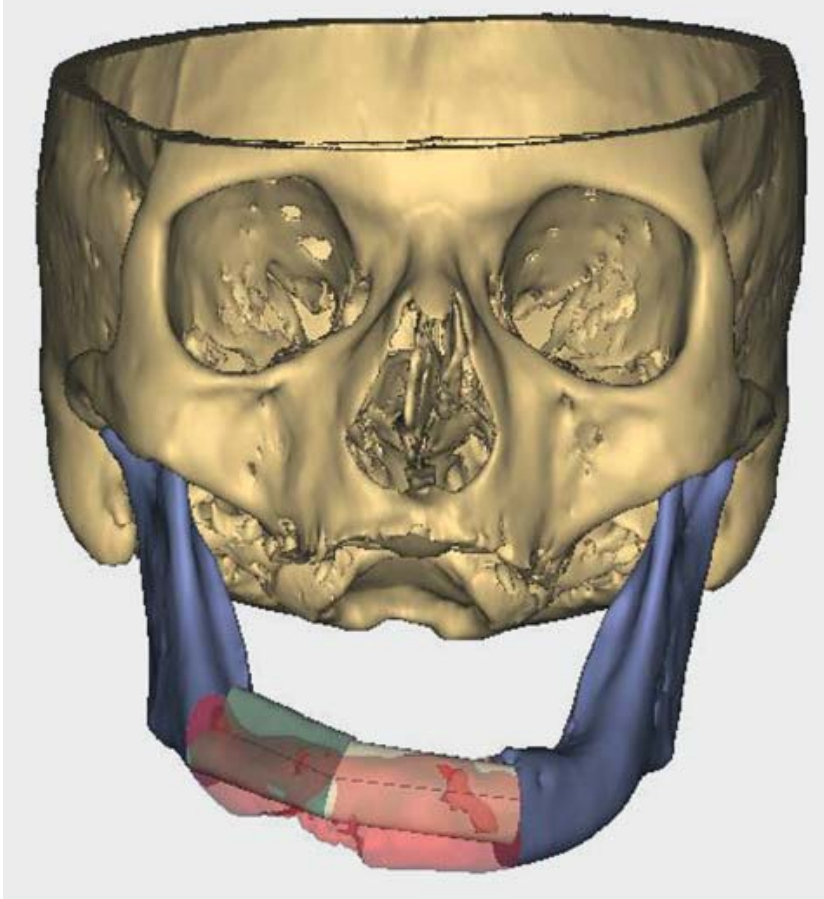

Abb. 2 Planung der Unterkieferrekonstruktion mittels 2 Fibulasegmenten (grün-grau) mit Überlagerung des resezierten Unterkieferanteils. aufgrund von Allgemeinerkrankungen wie insbesondere der pAVK vermindert werden und zu einer höheren Überlebensrate des Transplantats führen. Außerdem können bereits präoperativ Gefäßanomalien detektiert werden, was die Entscheidung des Operateurs bez. der Entnahmeregion von großer Hilfe sein kann. Bezüglich des vorliegenden klinischen Beispiels wurde als knöcherne Defektdeckung die Fibula gewählt.
Wenn alle notwendigen Datensätze vorliegen, werden diese anonymisiert über einen verschlüsselten FTP-Server der entsprechenden Produktionsfirma zugesendet. Diese erstellt aus den Datensätzen zunächst ein virtuelles anatomisches 3-D-Modell des entsprechenden Resektionsbereichs (Abb. 1) wie auch der Fibula (Abb. 3). Dann findet eine „Web-Konferenz" mit den behandelnden Chirurgen und den Ingeniueren der Produk- 

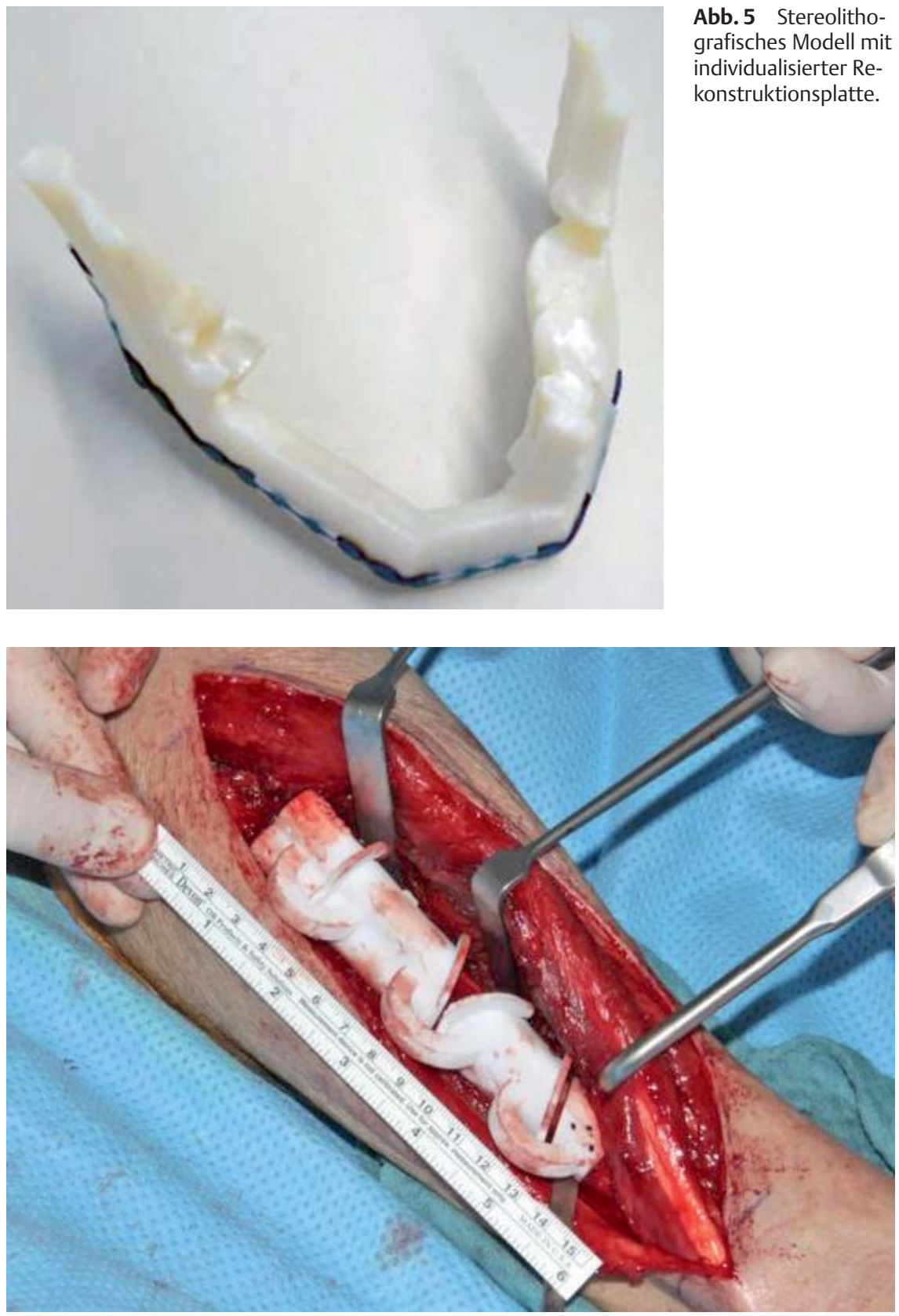

Abb. 6 In situ angebrachte Schablone zur exakten Resektion der Fibulasegmente.

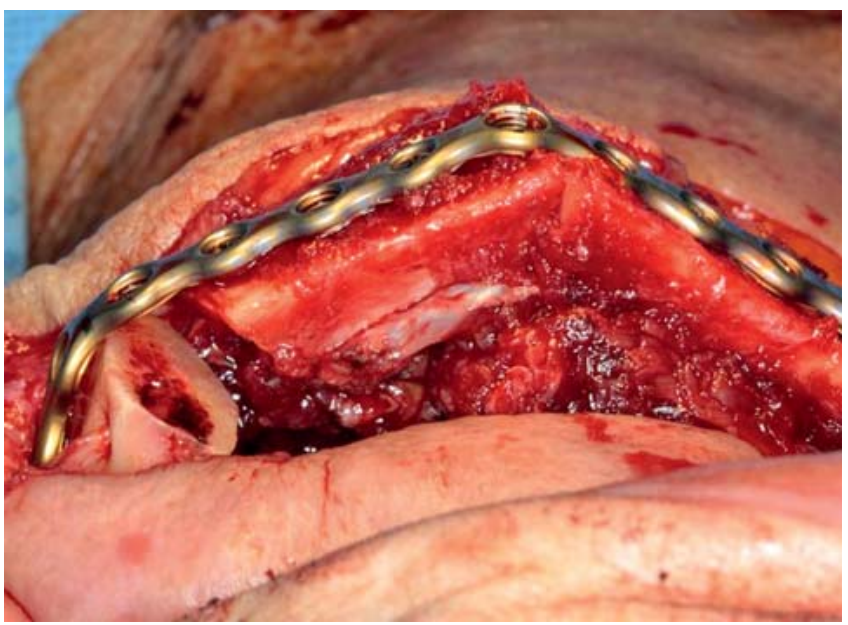

Abb. 5 Stereolithografisches Modell mit individualisierter Rekonstruktionsplatte. tionsfirma statt. Dabei werden die Resektionsgrenzen festgelegt, die hinterher durch die Produktion von passgenauen CAD-/CAM-gefrästen Sägeschablonen auf den operativen Situs übertragen werden (Abb. 1). Außerdem wird durch den Operateur in dieser Sitzung die genaue Lokalisation der Schnittgrenzen der Fibula und Anzahl der Segmente festgelegt, um postoperativ ein möglichst sowohl anatomisch als auch funktionell optimales Ergebnis zu erzielen. Auch dafür werden Schablonen angefertigt, die diese Informationen hinterher 1:1 auf den OP-Situs übertragen (Abb. 4).

Durch CAD-/CAM-Technik erstellte Sägeund Resektionsschablonen erleichtern dem Operateur die exakte Schnittführung.

Nach Festlegung der Positionierung des Gefäßstiels erfolgt in der Web-Konferenz die virtuelle Resektion des entsprechenden Kiefersegments, und die patientenspezifischen Fibulasegmente werden virtuell in das Modell mit eingefügt. Um dabei eine möglichst originalgetreue Rekonstruktion der ursprünglichen anatomischen Situation zu erreichen, wird das ursprünglich virtuell resezierte Segment überlagernd bildlich eingefügt, und es erfolgt die letzte Korrekturpositionierung der Fibulasegmente (Abb. 2). Nun erfolgt eine Festlegung des Operationstermins und die Produktionsphase beginnt. Darin wird anhand der zuvor virtuell erstellten Planungsmodelle ein 3-D-stereolithografisches Modell der postoperativen Situation (also nach Einsetzen der Fibulasegmente) erstellt. Anhand dieses realen Modells wird dann eine patientenspezifische Rekonstruktionsplatte angefertigt, die der postoperativen Situation (dargestellt an dem 3D-Planungsmodell) bez. Größe, Biegung, Anzahl der Bohrlöcher, angepasst wird (Abb.5). Ebenfalls in der Produktionsphase hergestellt werden die o.g. Bohrschablonen, die dem Operateur die Osteotomien im Kiefer und die Sägeschnitte in der Fibula ermöglichen. Insgesamt dauert die Produktionsphase ca. 14 Tage, dann werden dem behandelnden Chirurgen die Schablonen und die individuelle Rekonstruktionsplatte zugesendet, und es erfolgt die Operation.

Die CAD-/CAM-Technik ermöglicht eine exakte präoperative Planung und verbessert das funktionelle und ästhetische Outcome der Patienten. 


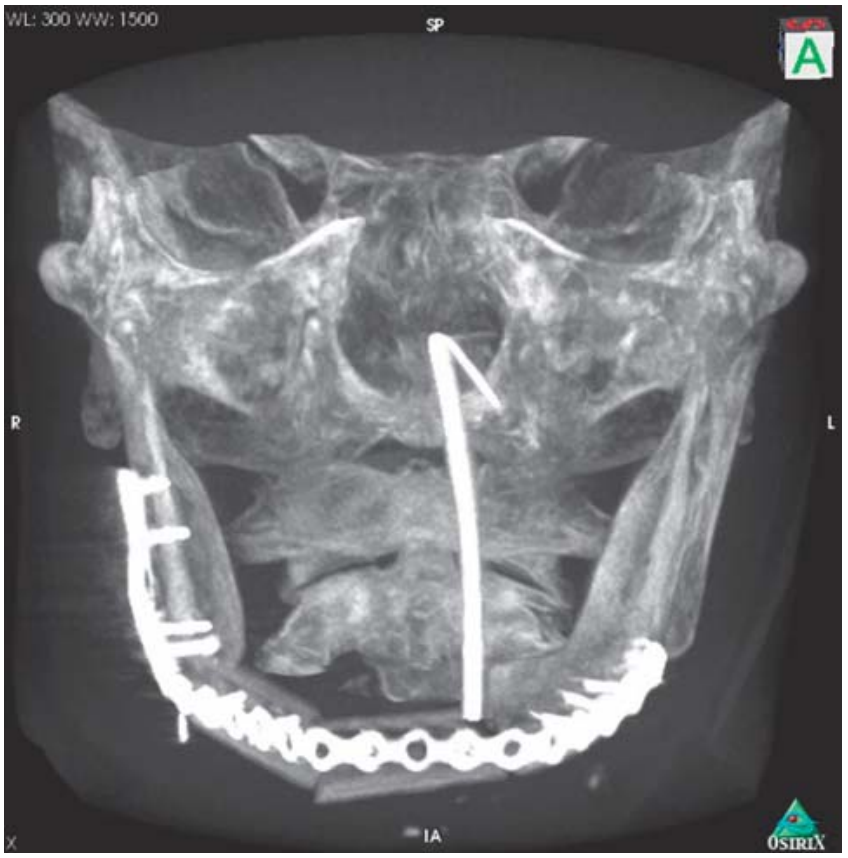

Abb. 8 Postoperatives Ergebnis.

Nach Darstellung der zu resezierenden Kieferabschnitte werden die Resektionsschablonen angebracht, sodass dem Operateur exakt definierte Osteotomieebenen vorgegeben sind (Abb.6). Gleiches Prozedere wird an der Fibula angewendet. Hier dienen die Schablonen einerseits der Definition der Absetzungsränder wie auch der Schnittführung der Segmente an sich. Im nächsten Schritt erfolgt die Hebung des Transplantats samt den versorgenden Gefäßen, und das Transplantat wird anhand der vorgefertigten individuellen Rekonstruktionsplatte (winkelstabil, Stärke 2.0; Abb. 7) im Kiefer des Patienten fixiert und mikrovaskulär anastomosiert. Die Operationsphase ist damit beendet, und es erfolgt im Verlauf die dentale Rehabilitation des Patienten (Abb. 8).
Dieses Verfahren hat sich mittlerweile als Standardverfahren etabliert, und seit seiner Ersteinführung 2011 wird allein an der Universitätsklinik Hamburg-Eppendorf jährlich eine Vielzahl an Patienten derartig behandelt.

Interessenkonflikt: Nein.

\section{Literatur}

${ }^{1}$ Hayden R, Mullin D, Patel A. Reconstruction of the segmental mandibular defect: current state of the art. Curr Opin Otolaryngol Head Neck Surg 2012; 20: 231-236

2 Antony A, Chen W, Kolokythas A et al. Use of virtual surgery and stereolithographyguided osteotomy for mandibular reconstruction with the free fibula. Plast Reconstr Surg 2011; 128: 1080-1084

3 Bell RB, Weimer KA, Dierks EJ et al. Computer planning and intraoperative navigation for palatomaxillary and mandibular reconstruction with fibular free flaps. J Oral Maxillofac Surg 2011; 69: 724-732

${ }^{4}$ Ciocca L, Mazzoni S, Fantini M et al. A CAD/ CAM-prototyped anatomical condylar prosthesis connected to a custom-made bone plate to support a fibula free flap. Med Biol Eng Comput 2012; 50: 743-749

${ }^{5}$ Ciocca L, Mazzoni S, Fantini M et al. The design and rapid prototyping of surgical guides and bone plates to support iliac free flaps for mandible reconstruction. Plast Reconstr Surg 2012; 129: 859-861

${ }^{6}$ Cohen A, Laviv A, Berman P et al. Mandibular reconstruction using stereolithographic 3-dimensional printing modeling technology. Oral Surg Oral Med Oral Pathol Oral Radiol Endodontol 2009; 108: 661-666

7 Foley BD, Thayer WP, Honeybrook A et al. Mandibular reconstruction using computeraided design and computer-aided manufacturing: an analysis of surgical results. J Oral Maxillofac Surg 2013; 71: e111-e119

Dr. med. Moritz A. Birkelbach

Wissenschaftlicher Mitarbeiter Dr. med. Christian Lohse

Wissenschaftlicher Mitarbeiter

Dr. med. Dr. med. dent.

Henning Hanken

Oberarzt

Priv.-Doz. Dr. med. Dr. med. dent.

Alexander Gröbe

Leitender Oberarzt und

stv. Klinikdirektor

Prof. Dr. med. Dr. med. dent.

Max Heiland

Klinikdirektor

Klinik und Poliklinik für Mund-, Kiefer- und Gesichtschirurgie Universitätsklinikum HamburgEppendorf

Martinistraße 52

20246 Hamburg

a.groebe@uke.de 